\title{
Medidas coercitivas en salud mental. Grupo focal con profesionales del ámbito comunitario
}

\author{
Coercive Measures in Mental Health Care. Focus Group with Professionals in the Field of Community Health
}

\author{
Carlos Aguilera Serrano ${ }^{1,2}$, Carmen Heredia Pareja ${ }^{3}$, José Guzmán Parra ${ }^{4}$, \\ Juan A. García Sánchez ${ }^{4}$, Fermín Mayoral Cleries ${ }^{4}$
}

\author{
'Universidad de Málaga, Andalucía Tech, Facultad de Psicología. Málaga, España. \\ ${ }^{2}$ USMC Motril, Área de Gestión Sanitaria Sur de Granada. Motril, España. \\ ${ }^{3}$ USMI-J, Hospital Regional Universitario de Málaga. Málaga, España. \\ ${ }^{4}$ Hospital Regional Universitario de Málaga. Instituto de Investigación Biomédica de Málaga (IBIMA). Málaga, España. \\ Contacto: c_aguiler2@hotmail.com \\ Fecha de recepción: 16 de septiembre de 2016 / Fecha de aceptación: 2 de diciembre de 2016
}

\begin{abstract}
Resumen
La coerción, con sus múltiples facetas, es el problema más antiguo de las instituciones psiquiátricas, principalmente por los numerosos intentos a lo largo de los tiempos por abolir y/o moderar su uso. La frecuencia, así como el marco jurídico que las regula, son extremadamente variados, lo que posibilita la transgresión de los derechos del paciente. Objetivo: Analizar la percepción de los profesionales de una Unidad de Salud Mental Comunitaria (USMC) ante la aplicación de medidas coercitivas hacia los usuarios que discurren por los distintos dispositivos de la Red de Atención a la Salud Mental en Andalucía.

Método: Se llevó a cabo un grupo focal con 10 participantes. El contenido del grupo focal fue transcrito y los temas fueron identificados usando análisis temático.

Resultados: Las categorías temáticas identificadas son: "Contexto conceptual sobre las medidas coercitivas", "Contexto situacional sobre las medidas coercitivas dentro del actual modelo de atención a la salud mental de Andalucía", "Medidas coercitivas como método de control", "Derechos humanos y empoderamiento de los usuarios" y "Sugerencias para el cambio".

Conclusiones: La utilización de medidas coercitivas parece estar plenamente justificada ante el actual modelo de atención y modos y medios de actuación. Se hace necesario invertir en salud mental, para el desarrollo de una mejora de infraestructura y servicios, así como una inversión en recursos humanos para proveer una atención y cuidados de calidad.
\end{abstract}

Palabras clave: análisis cualitativo, medidas coercitivas, unidades de salud mental comunitarias, intervenciones alternativas, práctica profesional.

\begin{abstract}
The coercion with its many dimensions, is the long-standing mental institutions problem, mainly because of the numerous attempts over the years to abolish and/or moderate the use of it. The frequency and the legal framework governing are extremely varied, which allows the infringement of patients rights.

Objective: To analyze the perception by professionals of a Community Mental Health Centers over the implementation of coercive measures toward the users that run along different devices which forms the mental health services in Andalucía.

Method: It took place a focus group of ten participants. The focal group content was transcribed and the topics were identified by using the thematic analysis.

Results: The thematic categories identified are: "The conceptual context on enforcement measure", "The situational context on enforcement measure within the actual model of attention or mental health care of Andalucía", "Coercive measures as a control method", "Human rights and empowerment of users" and "Suggestions for change."

Conclusions: The use of coercive measures seems to be fully substantiated request to the current model of activity. Investments in mental health are necessary to develop an improvement of infrastructure and services, and an investment in human resources to provide quality care are also necessary.
\end{abstract}

Keywords: qualitative analysis, coercion, community mental health centers, alternative interventions, professional practice. 


\section{Introducción}

Hoy en día, además de las intervenciones psicoterapéuticas y farmacológicas, el uso de la coacción a veces se considera como inevitable para controlar las desviaciones graves y/o perturbadoras del comportamiento/conducta que sufren aquellas personas aquejadas de algún problema de salud mental ${ }^{1}$. Sin embargo, la cuestión de la coerción, con sus múltiples facetas, es el problema más antiguo de las instituciones psiquiátricas, principalmente por los numerosos intentos a lo largo de los tiempos por abolir y/o moderar su uso ${ }^{2-4}$.

Se consideran formas coercitivas en el tratamiento psiquiátrico el ingreso involuntario, la retención tras un ingreso voluntario, la contención mecánica, el aislamiento y la administración forzosa de medicación. Estas son las medidas de mayor visibilidad, pero existen otras que tampoco pueden obviarse como la persuasión, la influencia interpersonal, la inducción o incluso la amenaza ${ }^{5}$.

El panorama en los distintos países europeos es extremadamente variado en lo que concierne a la frecuencia de tales medidas y el marco jurídico que las regula, lo que permite un amplio margen para que se infrinjan los derechos de los pacientes y no se apliquen las mejores prácticas clínicas ${ }^{6}$. Así, el Comité Europeo para la Prevención de la Tortura y de las Penas o Tratos Inhumanos o Degradantes (CPT) así como la Convención sobre los Derechos de las Personas con Discapacidad de la ONU exponen que las personas que sufren algún tipo de enfermedad mental grave han sido identificados como una población particularmente vulnerable a la violación de los derechos humanos ${ }^{7,8}$, insistiendo en la necesidad de prohibir definitivamente las contenciones mecánicas, el aislamiento o los tratamientos involuntarios.

La evidencia científica apunta y se inclina por exponer estrategias o nuevos mecanismos de gestión y cuidados que nos ayuden a moderar el uso de medidas coercitivas en el manejo y cuidados prestados hacia los pacientes en unidades de hospitalización psiquiátri$\mathrm{ca}^{9,10}$. Sin embargo, estas prácticas se siguen utilizando pese a los resultados que de las mismas se señalan, donde se refleja la expresión de una gama de respuestas negativas de terror o rabia, a sentirse despojados de su humanidad o pérdida de la dignidad en lo que respecta a la coerción percibida por los propios pacientes ${ }^{11-16}$. Los profesionales reiteran el alto grado de sufrimiento percibido por parte de sus usuarios, sin embargo, expo- nen la multitud de dificultades que se encuentra en cada contexto determinado, viéndose obligados a desplazarse entre la línea tan estrecha que separa el cuidado, control y seguridad hacia y por el paciente con la aplicación de algún tipo de medida coercitiva ${ }^{13,17}$.

Es destacable el hecho de que la mayor parte de los trabajos publicados exponen los comentarios y apreciaciones de profesionales del ámbito hospitalario, habiendo prácticamente una ausencia de trabajos donde los profesionales de las unidades de intervención comunitaria expongan sus apreciaciones en relación al uso y empleo de medidas coercitivas en salud mental. Este trabajo intenta dar voz a profesionales del ámbito comunitario $\mathrm{y}$, en concreto, exponer sus percepciones, vivencias y expectativas en relación al uso de las mismas.

\section{Objetivo}

Analizar la percepción de los profesionales que conforman el equipo de trabajo multidisciplinar de una Unidad de Salud Mental Comunitaria (USMC) ante la aplicación de medidas coercitivas hacia los usuarios que discurren por los distintos dispositivos que conforman la Red de Atención a la Salud Mental en Andalucía.

\section{Método}

La metodología empleada ha sido de tipo cualitativo, mediante la ejecución de un grupo focal, el cual nos permitió aportar información relevante sobre las percepciones que desarrollan los sujetos sociales en el proceso de interacción y de conversación con sus pares, dando la posibilidad así de revelar información sobre el nivel de conocimientos que tenían sobre el tema explorado ${ }^{18}$. Del mismo modo, nos permitió también observar cómo y por qué los individuos, en interacción, aceptan o rechazan las ideas de otros ${ }^{19}$ además de exponer la valiosa herramienta para la investigación exploratoria que supone este tipo de técnica, al permitirnos identificar temáticas que requieren ulterior profundización. Obviamente los participantes se conocían por lo que no se trataría de un grupo focal sensu stricto, pero pensamos que en el contexto que nos ocupaba esta circunstancia no invalidaba en absoluto la interacción de los individuos en el grupo ni, por tanto, la función de éste como método cualitativo. El resultado de la interacción verbal de los participantes en el grupo focal fue recogido con una grabadora para la posterior fase de interpretación y análisis de los datos. 
Se incluyeron 10 informantes ( 8 mujeres y 2 varones) de edades comprendidas entre 30 y 50 años, los cuales conforman y son miembros activos del equipo de trabajo de la Unidad de Salud Mental Comunitaria de Motril perteneciente al Área de Gestión Sanitaria Sur de Granada. Los perfiles profesionales representados por los participantes incluyen: técnico/a en Cuidados Auxiliares de Enfermería, diplomado/a en Enfermería, diplomado/a en Trabajo Social, facultativo/a especialista de Área Psiquiatría y Psicología Clínica, MIR de Psiquiatría.

No se determinaron criterios de inclusión o exclusión concretos, exceptuando la voluntariedad de participar en el estudio así como de acudir el día y hora preestablecida para su desarrollo. Se recabó la firma de todos los asistentes en relación al consentimiento informado, autorizando la grabación y posterior uso de la información para su análisis. No fue necesario incluir el permiso del Comité de Ética de Investigación ya que el trabajo no ha implicado recabar datos de población vulnerable (pacientes, familiares, niños, ancianos, etc.). El moderador puntualizó los aspectos de la confidencialidad e intimidad, no teniendo que llegar a profundizar en temas íntimos y personales, salvaguardando del mismo modo el anonimato mediante la codificación de cualquier tipo de dato identificativo.

La entrevista grupal tuvo una duración de 60 minutos y se realizó en la sala multiusos de la USMC, la cual, cuenta con amplias mesas colocadas en forma circular, lo que favoreció la comunicación digital y analógica entre los participantes. Para facilitar el diálogo, se partió de la siguiente batería de preguntas a modo de guía: ¿Qué conocéis sobre las medidas coercitivas? ¿Qué pensáis sobre su uso/empleo? ¿Hasta qué punto consideráis necesarias la aplicación de algún tipo de medida coercitiva? ¿Qué cosas, aspectos, situaciones, contextos, etc., pueden condicionar su uso? ¿Conocéis los efectos que para la salud puede tener? ¿Trabajáis o tratáis algún aspecto relacionado con su uso en la consulta? ¿Qué alternativas conocéis o propondríais para moderar/disminuir/eliminar su uso? ¿Qué se podría hacer desde las USMC en relación a este tema?

Finalmente, la entrevista grupal grabada en audio fue transcrita efectuándose a posteriori el análisis de contenido según las etapas descritas por Taylor Bogdan desarrollando categorías, codificando datos, separando éstos y finalmente, refinando el análisis ${ }^{20}$.

\section{Resultados}

El análisis del contenido reveló cinco categorías en relación con la percepción de los profesionales acerca de las medidas coercitivas en salud mental. A continuación se presentan las características centrales de las categorías, con ejemplos y transcripciones literales de la grabación.

1. Contexto conceptual sobre las medidas coercitivas (en base a sus propias ideas y experiencias así como del conocimiento y dominio teórico referido al tema de estudio).

Y.: Pues por medidas coercitivas quizás la que más se conoce sea la contención mecánica ¿no?... quizás por la gran repercusión mediática y por como lo violento de la misma...

C.J.: También el ingreso involuntario, o el uso de hipnóticos o medicación para el control conductual de los pacientes... también el aislamiento, aunque aqui por lo menos en Andalucía no hay espacios adecuados y habilitados para ello y serían buenos para no tener que llegar a utilizar a veces la contención mecánica...

M.: ... pues todas aquellas intervenciones que limitan o restringen la libertad y autonomía de la persona en situaciones en las que esta no tiene capacidad volitiva de actuar...

L.E.: ... todas aquellos tratamientos involuntarios y forzosos que utilizamos para el manejo de situaciones en las que por el estado del paciente o por el bien de terceros, es necesario instaurar...

Algunos participantes se muestran de acuerdo con estas medidas, creen que en ciertos casos son necesarias e incluso beneficiosas para la persona.

O.J.: ... cuando la medida se aplica de forma correcta, es porque las capacidades del paciente están alteradas de tal forma que se requiere el uso de la medida coercitiva como la contención pues está en riesgo su salud o la de terceros... el problema es cuando no se aplica de manera justificada, sino como mecanismo de opresión... para conseguir algo o como castigo.

Y.: Si de medida coercitiva estás hablando de no dar opción a un empeoramiento del paciente, sino a 
salvaguardad su seguridad el efecto es positivo, pues lo que se evita es el daño propio del paciente hacia sí mismo por su déficit de autocuidados o su estado de desestabilización psicopatológica.

C.J.: ... si las medidas son necesarias porque la situación y contexto del paciente lo requieran, considero que sí, que deben de aplicarse...la seguridad es primordial en casos extremos...

2. Contexto situacional sobre las medidas coercitivas dentro del actual modelo de atención a la salud mental de Andalucía. Algunos participantes posicionan de manera generalizada el uso de medidas coercitivas en entornos hospitalarios, concretamente, en las unidades de hospitalización de salud mental, mostrando dificultades en el reconocimiento de patrones coercitivos propios desde el ámbito comunitario. Sin embargo, todos reconocen la necesidad de establecer medidas legislativas que regulen su uso, pero sobre todo, una mayor inversión en recursos humanos, materiales e infraestructuras que ayuden en el progreso de mejora de los servicios y por ende, de la atención y cuidados especializados prestados.

C.J.: ... en el ámbito comunitario no se percibe mucho su uso ... salvo cuando se trata de tratamiento ambulatorio involuntario... pero eso aqui es raro... y es que además, el problema está en la legislación, pues no hay nada que regule... Los límites son dificiles de definir y por lo tanto, a veces no sabe uno bien ni cómo actuar...

M.: Independientemente de la regulación legislativa es necesario incidir en la necesidad de seguir dotando de recursos sociosanitarios al sistema que nos ayuden a garantizar una atención adecuada desde el entorno comunitario con pisos tutelados o programas de tratamiento asertivo comunitario... pues de lo contrario, es imposible que podamos avanzar...

Algo parece estar cambiando en los últimos años, pues perciben que en la actualidad hay más moderación en cuanto al uso de medidas coercitivas, y en concreto, sobre el uso de la contención mecánica. Todos identifican y reafirman la importancia de la relación terapéutica como pieza clave para el trabajo conjunto con el paciente, ya que en gran parte con lo que trabajamos es con la relación, que en sí misma es fuente de cuidados ${ }^{21}$. Sin embargo, uno de los problemas que se nos presentan es la influencia de lo personal en lo profesional, de cómo ésta influye en las observaciones y por extensión, en la intervenciones propuestas.

O.J.: Luego las medidas ya no son como antes... Cuando veíamos en las películas cuando se usaban camisas de fuerza y se tenía como preso al paciente, con tratamientos invasivos e invalidantes... Ahora las cosas han cambiado... son muy distintas y menos mal...

C.J.: ... de hecho, yo por ejemplo pienso que hay pocas contenciones o que se realizan menos en relación a las que podrían darse y gracias al tipo de relación que tenemos con nuestros pacientes... y es que yo pienso que un cincuenta por ciento del éxito clínico va a depender de establecimiento de una buena relación terapéutica o no...

Y.: Creo que somos una profesión, o mejor dicho, que los profesionales que nos dedicamos a esta área de la psiquiatría, trabajamos con aspectos subjetivos, que no se pueden explicar sólo o solamente por el fallo de un mecanismo determinado, como puede ser una obstrucción biliar... de hecho, incluso una valoración que yo pueda haber realizado de una persona en concreto puede ser que C.J. la valore y no vea los mismo y emita un juicio distinto... y es que, algo muy importante en nuestro modo de actuar es la relación de ayuda... ya no sólo como herramienta sino como medio para llegar al otro...

Los factores personales que influyen en la relación terapéutica (orientación teórica, uso del lenguaje y las variables sociológicas e individuales) son vitales para ayudar al paciente en su proceso de salud ${ }^{21}$. Los profesionales de enfermería son identificados por su trabajo diario y continuo junto al paciente, como principales influyentes en esta relación terapéutica, fuente principal de cuidados.

S.T.: Además tampoco se pretende ser poli malo o poli bueno, sino que se intenta trabajar las conductas, los comportamiento ... y claro... desde la consulta yo por ejemplo trabajo estos aspectos... sobre todo cuando ha sido un ingreso involuntario, explicando los motivos que me llevaron a adoptar esa medida, haciendo responsable al paciente de su comportamiento en un hecho de favorecer y trabajar la autoconciencia...

O.J.: Se le explica el por qué se ha hecho de esa mane$r a$, intentando sobre todo hacerle partícipe y res- 
ponsable de sus actos... es importante la explicaciones de cara a su autonomía, pues se le ayuda a conocerse más a sí mismo, a autocontrolarse, a saber pedir ayuda a su familia y sobre todo, a aprender a gestionar su proceso de enfermedad.

L.E.: ... además el tema de las contenciones casi depende más de enfermería que del psiquiatra, pues la gran mayoría de las veces cuando nos presenciamos, el evento ya ha sucedido, por lo que las decisiones ya han sido tomadas por la enfermera, teniendo tan sólo ya que firmar la prescripción de la misma...

C.J.: ... la enfermería de salud mental en general está mal valorada dentro de los compañeros de profesión del hospital... en el sentido de que no hacen grandes técnicas... sin embargo, pocos son los que hablan del poder de contención de la enfermería... ya sea en las unidad de agudos como en la comunidad en el domicilio del paciente... yo creo que no se imaginan la cantidad de horas que se pasan al lado del paciente hablando y trabajando sus miedos, ansiedades...

3. Medidas coercitivas como método de control. Los participantes discutieron cómo algunas medidas coercitivas, y en algunos contextos concretos, eran utilizadas por los profesionales para lograr el control de los pacientes así como también gestionar los entornos en los que se encontraban. Las siguientes citas captan la discusión acerca de cómo el control se refiere tanto a la conducta como al mantenimiento de las rutinas a fin de contener el medio ambiente.

A.M.: ... en las unidades de agudos existen como unas normas un poco particulares, donde todo parece ser que tiene que estar hecho de una manera concreta sin posibilidad de alternativas... por ejemplo, los horarios, todo tiene un horario, y muy estricto, por lo que ese simple hecho puede generar gran presión en determinadas personas... llegando a empeorar su estado.

C.J.: Claro, pero muchas normas de las que tú señalas son moldeadas por los propios profesionales según su beneficio... o ¿cuántas veces vemos en una unidad de agudos cómo los pacientes tienen medicación puesta para dormir por un tubo y a lo mejor eso no le hace falta porque el paciente duerme bien? ... pero por si acaso, se le deja prescrito...
A.M.: La rutina puede que ayude al control de la unidad... pero también la rutina es muy señalada por los propios pacientes, porque los días los pasan aburridos, y eso se nota nada más que te ven y quieren captar tu atención, hablar contigo, interactuar...

4. Derechos humanos y empoderamiento de los usuarios. En general, los participantes señalan las medidas coercitivas, y en concreto, a la contención mecánica, como una intervención que viola los derechos humanos del paciente, incluso cuando ésta pueda haber parecido necesaria para gestionar el riesgo. Además, su carga emocional no es unívoca sino que afecta a todos los implicados.

Y.: Desafortunadamente, creo que hay situaciones que requieren del uso de la contención mecánica porque verdaderamente es necesaria... pero por otro lado, esto atenta contra la libertad... incluso tal vez sea un trato indigno... es una cosa bastante dura para hacer para alguien... son situaciones complicadas $y$ dificiles donde hay que tomar decisiones muy apresuradas...

O.J.: ... pero debemos pensar en la carga emocional y sufrimiento aparejado a dicha situación, donde no sólo el paciente sufre... es que los profesionales lo pasanmal...

A.M.: Yo ha habido veces que tras un suceso, me he quedado pensando en si la forma de obrar ha sido la adecuada... o si hubiese cedido en aquello otro tal vez la situación hubiera ido por otra vía... no sé... es dificil tomar decisiones en esos momentos de tanta activación y tensión...

I.: ... el hecho de tener que actuar en contra de la voluntad del paciente circunscribe una serie de situaciones desagradables... No es de buen gusto ver sufrir a alguien... pero como decías, lo que prima es el bien del paciente...

Una persona inmersa en su proceso de recuperación es aquella que asume la responsabilidad de su propia salud mental en colaboración con la familia, los cuidadores y los propios profesionales sanitarios ${ }^{22}$. Además, el optimismo terapéutico por parte de los profesionales sanitarios es un factor importante en este sentido, pues el mismo se hace terapéutico al facilitar el procesamiento de la información negativa, dando lugar a formas completas y flexibles de apoyo y promoviendo el 
desarrollo del afrontamiento y resolución de proble$\operatorname{mas}^{23}$. En contraposición a estos argumentos, algunos participantes muestran una concepción algo escéptica sobre el proceso de empoderamiento, pues según su discurso, los sistemas de salud y la organización de los servicios son distintos de unos países a otros, lo que influye en la cartera de servicios y por extensión, en los modos de atención.

C.J.: No creo que todos los pacientes se puedan o deban empoderar dentro de la salud mental... ello requiere una capacidad de insight... de un autoconocimiento bastante importante, por lo que eso es una utopía... Hay pacientes con gran deterioro cognitivo que dificilmente pueden llegar a empoderarse como en los manuales viene reflejado... o por lo menos con nuestro modelo de atención... con eso no digo que perdamos el tiempo cuando los vemos...si no que trabajamos hacia el mantenimiento de las partes sanas, fortaleciendo sus decisiones... y con el tema de la coerción, pues pasa lo mismo... un paciente en fase aguda que abandona el tratamiento por falta de conciencia de enfermedad ¿qué? Pues no podemos permitirnos el lujo de poner en riesgo su salud... aunque en ese caso suponga una intromisión en su persona...

5. Sugerencias de cambio. Hay países donde no se practica la contención mecánica ${ }^{24}$ ya no sólo por aspectos legales, sino porque cuentan con sistemas y modelos de atención más orientados al ámbito comunitario, muy distintos al modelo de atención a la salud mental de Andalucía. Los participantes argumentan que con nuestro actual sistema difícilmente se pueda erradicar (que no moderar) el uso de la contención mecánica. Otras medidas como la administración de medicación forzosa son percibidas como menos traumáticas, e incluso, proponen como alternativa eficaz a la contención mecánica el aislamiento.

Y.: Yo creo que eso es inviable aqui en nuestro sistema (la contención mecánica)... porque no tenemos los recursos necesarios ni alternativas para trabajar y promover la seguridad del paciente agitado con riesgo de auto agredirse o agredir a un tercero...

A.M.: Yo en agudos considero necesario la creación de un espacio, una habitación habilitada de manera adecuada... de aislamiento, acolchada y vigilada... que ayudase a tranquilizar al paciente sin tener que llegar a usar la contención... de hecho, yo creo que eso ayudaría a disminuir su prevalencia...
Al preguntar sobre las posibles alternativas al uso de las medidas coercitivas en general, parecen no conocer las últimas evidencias sobre estrategias para reducir/moderar su uso ${ }^{10,25}$ aunque sí que exponen la sensibilización y formación de los profesionales como algo imprescindible.

S.T.: ... el hecho de crear espacios como este donde poder hablar del tema y sobre todo, exponer y discutir casos creo que sería el primer paso para trabajar sobre este tema con los profesionales.

A.M.: A los profesionales nos aburren las charlas directivas donde sólo se exponen directrices de cambio pero ahí se quedan ... sin aportar alternativas y mucho menos, sin trabajar las mismas... yo he ido a unas cuantas sesiones sobre contenciones mecánicas por ejemplo y siempre se habla y dicen lo mismo... que hay que disminuir, que se debe contener menos ... pero nada más, no explican formas de cambiar...

C.J.: Está bien informar ... pero trabajar con las consecuencias... si me dices que contenga menos por ejemplo, dame herramientas o enséñame alternativas...

A.M.: ... es verdad que ya tenemos instrumentos para trabajar... como la guía de decisiones anticipadas en salud mental ... pero es sólo eso... un instrumento ético pero sin valor legal... y por eso las políticas y la legislación debe de avanzar al mismo ritmo... pues de lo contrario, no podremos avanzar, sólo nos quedamos en la filosofía, en la teoría... y no en la práctica...

S.T.: Además son instrumentos que se utilizan en todos los niveles de atención, desde las unidades comunitaria hasta en el hospital, por lo que pueden ayudarnos a trabajar la gestión del riesgo desde todos los ámbitos de actuación...

Las opiniones son bastante variadas en relación a la necesidad de regular la práctica clínica con la existencia de protocolos de actuación sobre la utilización de medidas coercitivas. Algunos profesionales sanitarios se muestran más reacios a la idea porque creen que pueden limitar su autonomía de decisión, otros opinan que su existencia puede protegerlos, pues legitimaría sus actuaciones. Otros afirman que la existencia de un protocolo de actuación, como por ejemplo el establecido en Andalucía sobre Contención Mecánica ${ }^{26}$, podría 
incluso incrementar el uso de las medidas coercitivas, además de mostrar una actitud escéptica sobre el buen seguimiento y control del mismo en todo el territorio. Sin embargo, la mayoría consideran el establecimiento de protocolos $\mathrm{y} / \mathrm{o}$ procedimientos normalizados de trabajo como algo positivo y beneficioso tanto para los pacientes como para los profesionales.

Y.: ... con el actual modelo de atención es dificil decir que se puedan eliminar las medidas... claro que con el uso de los protocolos pues puede ser que ayude a moderar determinados aspectos... siempre bajo la ética de máximos que debemos cumplir...

\section{C.J.: Los protocolos deben de ayudarnos en la toma de} decisiones para dispensar una atención de salud mental eficaz. El problema tal vez sea que los mismos no se adapten a las circunstancias... o que no se evalúe su efectividad...

Identifican y relacionan el desarrollo de la ocupación en todos los ámbitos de actuación, tanto hospitalario como comunitario (no como una alternativa al uso de medidas coercitivas pero sí como una estrategia de prevención) con un mayor autoconocimiento y autocontrol emocional, lo que repercute en una aminorada necesidad de aplicar medidas coercitivas.

\section{C.J.: Hay pacientes que lo piden ... que le pongas medi-} camentos o que le ingreses o incluso los contengas... aunque son los que menos... pero hay pacientes que al estar ocupados, con un trabajo o una actividad, pues se notan más realizados, con mayor capacidad de control... y eso, pues repercute en el tipo de relación así como de intervenciones que se llevan a cabo con ellos.

A.M.: ... incluso en las unidades de agudos, la sala de terapia sirve como espacio de descarga... los pacientes andan más concentrados, relajados... y eso les ayuda a moderar sus impulsos.

\section{Conclusión}

No se puede interpretar que los datos expuestos en este trabajo reflejen la opinión y/o creencias de la mayoría de los profesionales que trabajan en las unidades de salud mental comunitaria, pues se hace necesario recordar que la metodología cualitativa no pretende nunca generalizar sus hallazgos. Simplemente, deben entenderse como opiniones representativas de este colectivo, los cuales, pueden aportar o servir para orientar nuevas líneas de investigación así como mejorar aspectos de la práctica clínica al reflexionar sobre lo expuesto.

En la opinión de los profesionales, la utilización de medidas coercitivas parece estar plenamente justificada por la dificultad en el manejo de episodios de auto o heteroagresión, como recurso para amparar la seguridad clínica y, en definitiva, como métodos para salvaguardar el estado de salud de los usuarios. Todos coinciden en señalar la necesidad de inversión en salud mental, ya no sólo financiera, la cual fuese designada para el desarrollo de una mejora de infraestructura y servicios sino también, una inversión en recursos humanos para proveer una atención y cuidados de calidad que incorporen procedimientos adecuados que protejan los derechos de las personas con algún problema de salud mental.

\section{Bibliografía}

1. Steinert T, Noorthoorn EO, Mulder CL. The use of coercive interventions in mental health care in Germany and the Netherlands. A comparison of the developments in two neighboring countries. Front public Heal [Internet]. 2014 Jan [cited 2014 Dec 17];2(September):141. Available from:

http://www.pubmedcentral.nih.gov/articlerender.fcgi?artid=417 3217\&tool=pmcentrez\&rendertype=abstract

2. Amico L del C. Desmanicomialización: "Hacia una transformación de los Dispositivos Hegemónicos en Salud Mental" [Internet]. Periódico de Trabajo Social y Ciencias Sociales Edición electrónica. 2004. Available from: http://www.margen.org/suscri/margen35/amico.html

3. Steinert T, Lepping P, Bernhardsgrütter R, Conca A, Hatling T, Janssen $W$, et al. Incidence of seclusion and restraint in psychiatric hospitals: a literature review and survey of international trends. Soc Psychiatry Psychiatr Epidemiol [Internet]. 2010 Sep [cited 2013 Aug 12];45(9):889-97. Available from: http://www.ncbi.nlm.nih.gov/pubmed/19727530

4. Martin V, Bernhardsgrütter R, Goebel R, Steinert T. Clinical Practice and Epidemiology The use of mechanical restraint and seclusion in patients with schizophrenia : A comparison of the practice in Germany and. 2007;6:1-6.

5. Busch AB. Seclusion and restraint: a review of recent literature. Harv Rev Psychiatry. 2000;8(5)(2):261-70.

6. Mayoral, F. , Torres, Francisco GE. La utilización de medidas coercitivas en psiquiatría. Actas Españolas Psiquiatr. 2005;33(5):331-8.

7. United Nations. Report of the Special Rapporteur on torture and other cruel, inhuman or degrading treatment or punishment [Internet]. 2013 [cited 2016 May 3]. Available from: http://www.refworld.org/docid/51136ae62.html

8. Consejería de Salud y Bienestar Social. Junta de Andalucía. Derechos Humanos y Salud Mental en Andalucía. 2012.

9. Guzman-Parra J, Garcia-Sanchez JA, Pino-Benitez I, Alba-Vallejo M, Mayoral-Cleries F. Effects of a regulatory protocol for mechanical restraint and coercion in a Spanish psychiatric ward. Perspect Psychiatr Care. 2014; at press.

10. National Association of State Mental Health Program Directors. Six Core Strategies for Reducing Seclusion and Restraint Use (c) [Internet]. 2006 [cited 2014 Nov 1]. Available from: http://webcache.googleusercontent.com/search?q=cache:7Nq kVJxtsvkJ:www.nasmhpd.org/docs/NCTIC/Consolidated_Six_ 
Core_Strategies_Document.pdf $+\& c d=2 \& h l=e s \& c t=c \mid n k \& g l=e s$ 11. Brophy LM, Roper CE, Hamilton BE, Tellez JJ, McSherry BM. Consumers and Carer perspectives on poor practice and the use of seclusion and restraint in mental health settings: results from Australian focus groups. Int J Ment Health Syst [Internet]. BioMed Central; 2016;10:6. Available from: http://www.pubmedcentral.nih.gov/articlerender.fcgi?artid $=474$ 4440\&tool=pmcentrez\&rendertype $=$ abstract

12. Lanthén K, Rask M, Sunnqvist C. Psychiatric Patients Experiences with Mechanical Restraints: An Interview Study. Psychiatry J [Internet]. 2015;2015:748392. Available from: http://www.pubmedcentral.nih.gov/articlerender.fcgi?artid=449 6488\&tool=pmcentrez\&rendertype=abstract

13. Holmes D, Murray SJ, Knack N. Experiencing Seclusion in a Forensic Psychiatric Setting: A Phenomenological Study. J Forensic Nurs [Internet]. 2015;11(4). Available from:

http://www.ncbi.nlm.nih.gov/pubmed/26457901

14. Kontio R, Joffe G, Putkonen H, Kuosmanen L, Hane K, Holi M, et al. Seclusion and restraint in psychiatry: patients' experiences and practical suggestions on how to improve practices and use alternatives. Perspect Psychiatr Care [Internet]. 2012 Jan [cited 2014 Nov 14];48(1):16-24. Available from: http://www.ncbi.nlm.nih.gov/pubmed/22188043

15. Nelstrop L, Chandler-Oatts J, Bingley W, Bleetman T, Corr F, CroninDavis $J$, et al. A systematic review of the safety and effectiveness of restraint and seclusion as interventions for the short-term management of violence in adult psychiatric inpatient settings and emergency departments. Worldviews Evid Based Nurs [Internet]. 2006 Jan [cited 2014 Nov 23];3(1):8-18. Available from: http://www.ncbi.nlm.nih.gov/pubmed/17040518

16. Girela E, López Á, Ruiz F. Estudio de las medidas coercitivas en centros penitenciarios y hospitales psiquiátricos penitenciarios : opiniones de internos y profesionales. 2014;3-10.

17. Holmes D, Kennedy SL, Perron A. the Mentally III and Social Exclusion: a Critical Examination of the Use of Seclusion From the Patient'S Perspective. Issues Ment Health Nurs [Internet]. 2004;25(6):559-78. Available from:

http://www.tandfonline.com/doi/full/10.1080/0161284049047 2101

18. Liamputtong P. Focus Group Methodology : Introduction and History. Qual Res Methods. 2010;1-14.

19. Steward D, Shamdasani P RD. Focus Groups. Theory and Practice. 2a ed. California: Thousand Oaks; 2007.

20. Bodgan T. Introducción a los métodos cualitativos de investigación. Barcelona: Paidos; 1998.

21. García Laborda A, Rodríguez Rodríguez JC. Factores personales en la relación terapéutica. Rev la Asoc Española Neuropsiquiatría. 2005;XXV(29):29-36.

22. Nicholas Procter HP, Hamer D, McGarry RL, Froggatt $W$ and T. Mental health a person-centred approach. Cambridge, editor. Australia; 2014.

23. Byrne MK, Sullivan NL, Elsom SJ. Clinical optimism: Development and psychometric analysis of a scale for mental health clinicians. Aust J Rehabil Couns [Internet]. 2006 [cited 2016 May 8];12(1):11-20. Available from: http://ro.uow.edu.au/cgi/viewcontent.cgi?article=1153\&context =hbspapers

24. Stewart D, Bowers L, Simpson A, Ryan C, Tziggili M. Manual restraint of adult psychiatric inpatients: a literature reviw. J Psychiatr Ment Health Nurs. 2009;16:749-57.

25. Bowers L. Safewards: a new model of conflict and containment on psychiatric wards. J Psychiatr Ment Health Nurs [Internet]. 2014 Aug [cited 2014 Dec 17];21(6):499-508. Available from: http://www.pubmedcentral.nih.gov/articlerender.fcgi?artid $=423$ 7187\&tool=pmcentrez\&rendertype=abstract

26. Servicio Andaluz de Salud. Consejería de Salud. Protocolo de Contención Mecánica. Consejería de Salud y Bienestar Social. Junta de Andalucía., editor. Sevilla; 2010. 